\title{
Equipment
}

\section{Contamination suscepti- bility of three needleless and one standard needle injection systems}

Anthony M.-H. Ho MD MSC FRCPC, Elizabeth Ling MD MSC FRCPC

Purpose: To compare in vitro the contamination susceptibility of three needleless and one standard needle injection systems.

Methods: The Baxter InterLink, St. Paul Medical Key-Lok, Braun SafSite, and Becton

Dickinson heparin lock, were compared. Fifty of each were studied. Each system was connected to a culture bottle and injected 30 times over $72 \mathrm{hr}$. The manufacturers' directions were followed except for Braun's, which recommend that a new sterile cap be used after each injection. Instead, the same cap was used after each injection to reflect day-to-day clinical practice. Culture bottles were subsequently analyzed for bacterial growth.

Results: The contamination rates for the heparin lock, InterLink, Key-Lok, and SafSite were 3/50 (6\%), 6/50 (12\%), 4/50 (8\%), and 36/50 (72\%), respectively. All positive cultures grew coagulase-negative staphylococcus species. There was no difference in contamination rates between the InterLink, Key-Lok, and heparin lock designs. The contamination rate of the SafSite was higher $(P<0.001)$ than the other three systems.

Conclusions: The InterLink and Key-Lok systems had a similarly low contamination susceptibility as a standard needle injection system. It was not clear if the high contamination rate found with the SafSite system was due to non-adherence to the manufacturer's directions for handling.

Objectif : Comparer in vitro la susceptibilité à la contamination de trois appareils d'injection sans aiguille et d'un standard avec aiguille.

Méthode : Les dispositifs suivants ont été comparés : le Baxter InterLink, le St. Paul Medical Key-Lok, le Braun SafSite, et le Becton Dickinson heparin lock. On en a étudié 50 dans chaque catégorie. Chaque dispositif était relié à un flacon de culture et recevait 30 injections pendant $72 \mathrm{~h}$. Les instructions du manufacturier ont été suivies, sauf pour le Braun qui recommandait qu'une nouvelle capsule stérile soit utilisée après chaque injection. On a plutôt réutilisé la même capsule comme dans la pratique clinique quotidienne. Les flacons de culture ont été analysés par la suite pour vérifier la croissance bactérienne.

Résultats : Les taux de contamination pour l'heparin lock, l'InterLink, le Key-Lok et le SafSite ont été de 3/50 (6 $\%), 6 / 50(12 \%), 4 / 50(8 \%)$, et de $36 / 50(72 \%)$, respectivement. Toutes les cultures positives ont mis en évidence la présence de staphylocoques à coagulase négative. II n'y avait pas de différence de taux de contamination entre l'InterLink, le Key-Lok et l'heparin lock. Le taux de contamination du SafSite a été plus élevé $(P<0,001)$ que celui des autres dispositifs.

Conclusion : L'InterKink et le Key-Lok présentaient une faible susceptibilité à la contamination, semblable à l'appareil standard avec aiguille. On ne peut être sûr que le haut taux de contamination du SafSite soit lié au non respect des instructions de manipulation du manufacturier.

From the Department of Anaesthesia, Hamilton Health Sciences Corporation and McMaster University Faculty of Health Sciences, Hamilton, Ontario.

Address correspondence to: Dr. Anthony Ho, Department of Anaesthesia and Intensive Care, Prince of Wales Hospital, Shatin, NT, Hong Kong. Phone: 852-2632-2735; Fax: 852-2637-8010; E-mail: hoamh@hotmail.com

Presented, in part, at the Canadian Anaesthetists' Society Annual Meeting in June 1997, Vancouver, British Columbia.

Supported by a grant from the Physicians Services' Incorporated Foundation, Toronto, Ontario. The needleless systems were supplied free-of-charge by their manufacturers.

Accepted for publication November 30, 1998 


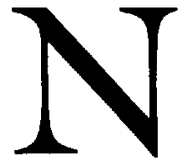

EEDLESTICK injury among health care workers ${ }^{1-4}$ is serious given the incidence of HIV and hepatitis virus infections in the population. Exposure among these workers is associated with transmission rates of $0.4 \%$ for $\mathrm{HIV}$ and $10-35 \%$ for hepatitis B virus, ${ }^{2}$ with most transmissions originating from needlestick exposures. Puro et al. ${ }^{5}$ found that hollow-bore needlesticks resulted in a $1.2 \%$ incidence of seroconversion of hepatitis $\mathrm{C}$ virus when the needles had been exposed to infected patients. Testing and counselling after each needlestick exposure cost US $\$ 450$, higher if prophylaxis for HIV is needed, and much higher if transmission of disease occurs. ${ }^{6}$

In response, a growing number of needleless systems are available. Most have latex or synthetic ports that are pre-slit or pre-drilled. Injections via these ports are done with blunt cannulas. A two-way valve design that allows direct injection with a syringe is also available. It is not clear whether these systems have different susceptibility to microbial contamination through the pre-formed ports, when compared with standard needle injection ports.

The purpose of this study was to compare the contamination susceptibility of three needleless and one standard needle injection systems under identical conditions and independent of patient factors.

\section{Methods}

The three needleless systems studied were the InterLink (Baxter Healthcare Corporation, Intravenous Systems Division, Deerfield, IL), Key-Lok (St. Paul Medical, Minneapolis, MN), and SafSite (B. Braun Medical Inc., Bethlehem, PA), against a standard heparin lock (PRN Adaptor, Becton Dickinson Vascular Access, Sandy, UT). The InterLink system has a latex septum with a resealable slit. The Key-Lok system has a synthetic septum, made of an elastomeric material, with a resealable hole. The SafSite has a two-way valve. The heparin lock septum is made of latex through which needles are inserted.

The study used 50 InterLink vial adaptors, 50 KeyLok vial adaptors, 50 SafSites, and 50 heparin locks. The latex diaphragm of a Baxter Bactec high volume resin aerobic blood culture bottle was punctured with an adaptor/injection port. The InterLink and KeyLok adaptors had an injection port at one end, and a piercing pin at the other, whereas the SafSites and heparin locks needed the attachment of an 18 gauge hypodermic needle. There were a total of 200 injection port-culture bottle sets. Each port was injected 30 times over $72 \mathrm{hr}$ (see below). The InterLink and Key-Lok ports were injected with the companies' own injection cannulas, the SafSites directly from $3-\mathrm{ml}$ syringes, and the heparin locks with standard 1.25 inch 18 gauge hypodermic needles. A new sterile injector was used for each injection. Each injection of $0.25 \mathrm{ml}$ of normal saline was drawn with a new sterile 3 - $\mathrm{ml}$ syringe from a new sterile bottle of $10-\mathrm{ml}$ normal saline. An experienced critical care nurse, wearing non-sterile but clean latex gloves, in a regular but vacant room (with minimal traffic) in an intensive care unit performed the entire experiment.

Prior to each injection of an individual port, it was swabbed with a new 70\% isopropyl alcohol swab according to the manufacturer's guidelines. In keeping with common practice, the injections were performed immediately after swabbing. According to B. Braun Medical Inc., the SafSite requires no alcohol swabbing. All SafSites were capped between injections. Although B. Braun recommends that a new sterile SafSite cap be used after each injection, in reality most practitioners recap with the same cap and the experiment was thus carried out using the same cap for each SafSite throughout the $\mathbf{7 2} \mathrm{hr}$.

All culture bottles were arranged inside a large box in a sequence of A-B-C-D-A-B-C-D-A... such that no two same types of injection ports were adjacent to each other and the different designs were equally distributed throughout the box.

Since the task of injecting 200 ports 30 times each was very time-consuming, the experiment was divided into two identical and consecutive sessions. In the first session, 25 of each of the four designs ( 100 port-culture bottle sets in total) were injected. During the first $12 \mathrm{hr}$, ten injections were made to each of the 100 port-culture bottle sets. The sets were then left exposed (except for the SafSites, which were capped) at the same location in the intensive care unit for the next $12 \mathrm{hr}$. The next morning, ten injections were made to each of the 100 port-culture bottle sets over $12 \mathrm{hr}$, followed by $12 \mathrm{hr}$ of rest. This protocol was repeated on the third day. Seventy-two hours after the commencement of the first injection, the culture botthes (without the injection ports) were sent for microbiologic analysis. All culture bottles were coded to blind the type of port system with which it was paired. The injections were performed in the following sequence: brand A-B-C-D-A-B-C-D-A..., such that the injections were all spread out equivalently over time for each design.

The second session was identical to the first and was performed by the same study nurse a week later using a new batch of 100 port-culture bottle sets ( 25 of each of the four designs). In addition, the tops of the 25 SafSite valves used in the second session were swabbed at the end of the $72 \mathrm{hr}$ period and sent for 
bacterial analysis. This was done because the preliminary results of the first session revealed a high contamination rate associated with the SafSite. The study nurse remained blinded from the preliminary culture results. The bacterial analysis of the SafSite in the second session was different from the analysis of the culture bottles that were attached to the injection ports.

All culture bottles, with the injection ports removed after $72 \mathrm{hr}$, were incubated at $35^{\circ} \mathrm{C}$ in an ambient air incubator after addition of Fastidious Organism Supplement. The bottles were subcultured after 18-24 hr of incubation, and again after $72 \mathrm{hr}$ of incubation, and finally after five days of incubation. The bacterial types were identified but antibiotic susceptibility was not tested.

The contamination rates between independent samples were analyzed using $\chi^{2}$ test with significance set at 0.05 .

\section{Results}

The number of positive cultures found with the injections systems (50 of each kind) were: Heplock: 3, InterLink: 6, Key-Lok: 4, and SafSite: 36 . The rates of contamination between the four systems were found to be different $\left(\chi^{2}(\mathrm{df}=3)=81.82, P<0.001\right)$. Since SafSite stood out conspicuously as highly contaminated, it was compared with all of the other systems combined and found to have a higher contamination rate $\left(\chi^{2}(\mathrm{df}=1)=77.93, P<0.001\right)$. The overall $\chi^{2}$ analysis without SafSite showed that there was no difference in contamination rates among the heparin lock, InterLink, and Key-Lok systems $\left(\chi^{2}(\mathrm{df}=2)=1.188, P>0.01\right)$.

All 49 positive cultures grew coagulase-negative staphylococcus species except one, which also grew a streptococcus species. The results suggest contamination by skin flora. All 25 swabs of the SafSite valves involved in the second half of the experiment failed to culture any bacteria. Nineteen of the 36 SafSites whose culture bottles grew bacteria came from this group.

\section{Discussion}

The finding of equivalent and low contamination rates with the InterLink and Key-Lok systems, compared with a standard needle injection system, suggests that these two systems may provide protection to health care workers from needlestick injuries without increasing the incidence of line contamination.

The incidence of intravenous (iv)-related infection among patients is $2-5 \%{ }^{7}$ This rate is influenced by the type of iv device, infusates, duration of iv placement, patient conditions, ${ }^{8,9}$ and probably the number of injections performed. Prospectively, Adam $e t a l .{ }^{10}$ compared the InterLink system with a conventional needle system (heparin lock). No difference ( $40 \% v s 48 \%$, respectively) in the incidence of $i v$ infection indicators such as site erythema, induration and tenderness, fever, or positive catheter or adaptor fluid culture was found. The high incidences of such indicators they found might have been influenced by the fact that $96 \%$ of their subjects were transplant or diabetic patients.

The finding of a high contamination rate with the SafSite design was disturbing. SafSite had been found to be no different from heparin lock in allowing the persistence of inoculated microorganisms in one study. ${ }^{11}$ The factors accounting for the high contamination rate of SafSite in our study are unknown but may, in part, be due to the lack of alcohol swabbing prior to each injection, and the presence of deep grooves in the SafSite injection ports. Although half of the SafSites were swabbed superficially and had no bacterial growth, it is possible that fluid may collect in the deep groves and become a reservoir for bacterial growth. More importantly is that contamination might have occurred during the extensive amount of handling (removing and recapping with every injection) of the Safsite caps. Even though much care was exercised to avoid contamination, the extensive handling during the experiment was not different from real clinical conditions. It is not clear if the contamination rate of the SafSite would have been lower had the manufacturer's recommendation of recapping with a new cap after each injection been followed. Further study is required.

In conclusion, two of the needleless injection systems tested were found to have a low contamination susceptibility to bacterial contamination upon multiple injections over $72 \mathrm{hr}$ in an intensive care unit. Their rates of contamination were no different from that of a standard needle injection system. The proper use of the Key-Lok and InterLink needleless systems in intravenous sites and multidose medication vials may not result in increased bacterial contamination compared to the standard needle injection ports commonly used today. The SafSite, when tested without following the manufacturer's direction of using a new sterile cap after each injection, was associated with a high incidence of contamination.

\section{References}

1 Marcus $R$ and the CDC Cooperative Needlestick Surveillance Group. Surveillance of health care workers exposed to blood from patients infected with the human immunodeficiencey virus. N Engl J Med 1988; 319: 1118-23.

2 Berry AJ, Greene ES. The risk of needlestick injuries and needlestick-transmitted diseases in the practice of anesthesiology. Anesthesiology 1992; 77: 1007-21. 
3 Greene ES, Berry AJ, Arnold WP III, Jagger J.

Percutaneous injuries in anesthesia personnel. Anesth Analg 1996; 83: 273-8.

4 Gerberding $J L$. Current epidemiologic evidence and case reports of occupationally acquired HIV and other bloodborne diseases. Infect Control Hosp Epidemiol 1990; 11: 558-60.

5 Puro V, Petrosillo N, Ippolito G. Risk of hepatitis C seroconversion after occupational exposures in health care workers. Am J Infect Control 1995; 23: 273-7.

6 Armstrong $S E$. The cost of needle-stick injuries: the impact of safer medical devices. Nursing Economy 1991; 9: 426-30, 433.

7 Maki DG, Weise CE, Sarafin HW. A semiquantitive culture method for identifying intravenous-catheterrelated infection. N Engl J Med 1977; 296: 1305-9.

8 Tager TB, Ginsberg MB, Ellis $S E$, et al. An epidemiologic study of the risks associated with peripheral intravenous catheters. Am J Epidemiol 1983; 118: 839-51.

9 Bostrom-Ezrati J, Dibble S, Rizzuto C. Intravenous therapy management: who will develop insertion site symptoms? Appl Nurs Res 1990; 3: 146-52.

10 Adams $K S, Z$ Zebrer CL, Thomas $W$. Comparison of a needleless system with conventional heparin locks. Am J Infect Control 1993; 21: 263-9.

11 Larson $E L$, Cheng $G$, Choo JTE, Merz W. In vitro survival of skin flora in heparin locks and needleless valve infusion devices. Heart Lung 1993; 22: 459-62. 